\title{
Programa de reciclaje electrónico en Ingeniería Electrónica de la cun
}

Jessica Rodríguez Velásquez*, Stefany Zuluaga Zubieta**, Daniela Mosquera Osuna***

\section{Resumen}

Este artículo tiene la finalidad de socializar a todas las universidades que ofertan programas de ingeniería electrónica, el desarrollo de un programa de reciclaje electrónico. Este partió como una iniciativa del semillero de investigación ElectroCUN que busca hacer frente al gran problema de la contaminación del medio ambiente por el pésimo manejo de los residuos eléctricos y electrónicos, habitualmente tratados como basura común. En este trabajo se detallan algunas empresas colombianas que han asumido esta misión, pero se nota necesario que en las universidades también se practique el reciclaje electrónico, actividad beneficiosa para las instituciones, los estudiantes y el ecosistema.

Palabras clave: artesanías basadas en residuos electrónicos, reciclaje, recuperación, residuos de aparatos eléctricos y electrónicos (RAEE)

\section{Abstract}

This paper aims to socialize to all universities that offer electronic engineering programs, the developing of an electronic recycling program. This started as an initiative of the ElectroCUN research group, that seeks to tackle the big problem of environmental pollution by the poor management of electrical and electronic waste, usually treated as common waste. This paper details some Colombian companies that have assumed this mission, to point out the need for universities to also practice e-recycling, a beneficial activity for institutions, students and the ecosystem.

Keywords: Waste Electrical and Electronic Equipment (WEEE), Recycling, Recovery, Electronic Waste-Based Handicraft.

\footnotetext{
* Estudiante del programa de Ingeniería Electrónica de la Corporación Unificada Nacional de Educación Superior (CUN). Contacto: jessica.rodriguezve@cun.edu.co

** Estudiante del programa de Ingeniería Electrónica de la Corporación Unificada Nacional de Educación Superior (CUN). Contacto: stefanny.zuluaga@cun.edu.co

${ }^{* * *}$ Estudiante del programa de Ingeniería Electrónica de la Corporación Unificada Nacional de Educación Superior (CUN). Contacto: daniela.mosquera@cun.edu.co
} 


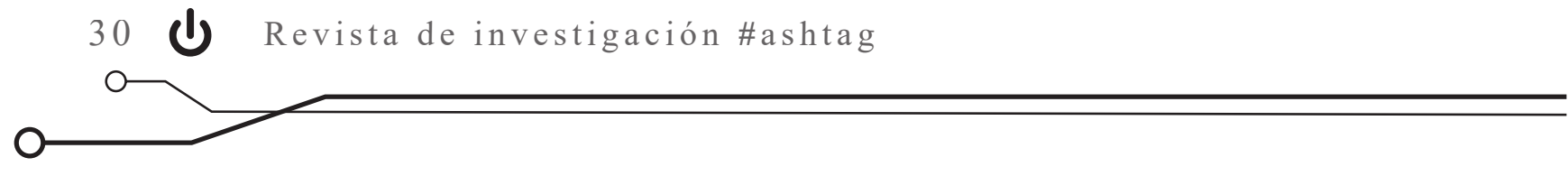

\section{Introducción}

Los residuos electrónicos son un gran problema para el medio ambiente por su corrosividad, reactividad, explosividad, toxicidad, inflamabilidad, o porque contienen sustancias dañinas que los hacen potencialmente peligrosos. Por ende, es preciso implementar programas locales de reciclaje electrónico que manejen dichos residuos y así evitar la contaminación producida por los elementos químicos que expulsan en el transcurso del tiempo.

El progreso tecnológico ha aumentado cada vez más y, con el pasar del tiempo, también aumentan más los residuos electrónicos. Por eso, algunas empresas han decidido enfrentar este problema recolectando particularmente artefactos que ya no se usan en los hogares o que, con el pasar el tiempo, se han vuelto obsoletos.

\section{Reciclaje electrónico}

En la actualidad, muchas personas aún desconocen que los electrodomésticos y aparatos electrónicos se pueden reciclar o que tienen componentes reciclables. De algunos de ellos se pueden extraer y almacenar sus tarjetas electrónicas con el propósito de reutilizarlas en la reparación de otros dispositivos.

Una manera de lograr que las personas tomen conciencia y enfrenten el potencial impacto negativo de dichos aparatos en el medio ambiente es enseñarles algunas técnicas sencillas de este
Actualmente, en el programa de Ingeniería Electrónica de la cun no se ha establecido ningún procedimiento de reciclaje para equipos obsoletos, ni para reciclar los componentes electrónicos que pueden obtenerse de artefactos eléctricos o electrónicos que ya no se usan o que ya han finalizado su vida útil de funcionamiento. Frente a dicha situación, en el semillero de investigación ElectroCUN se está considerando una posible alternativa que beneficiaría a toda la comunidad del programa de Ingeniería Electrónica de la institución: disponer estratégicamente de los residuos eléctricos y electrónicos, es decir, establecer un sistema de recolección, evaluación y clasificación de dichos residuos, con el propósito de organizarlos y promover su uso tanto en aplicaciones electrónicas como en otras instancias ajenas a la electrónica.

tipo de reciclaje o, al menos, indicarles a dónde llevar los aparatos que ya no son utilizados para su apropiado reciclaje. Los aparatos eléctricos y electrónicos son cada vez más numerosos en nuestras vidas y, en general, pueden ser agrupados en pequeños y grandes electrodomésticos, computadores, equipos de radio y comunicaciones, aparatos electrónicos enfocados al entretenimiento, dispositivos de iluminación, herramientas eléctricas, equipos deportivos, equipos médicos, instrumentos electrónicos y máquinas expendedoras (Velásquez, 2016). 


\section{Reciclaje de aparatos eléctricos y electrónicos (AEE)}

Los ciudadanos concienciados con el medio ambiente deberían aplicar las tres erres de la ecología (Responsabilidad Social Empresarial y Sustentabilidad, 2014) en el contexto de los aparatos eléctricos y electrónicos, así:

Reducir: disminuir la cantidad de AEE, empleados tanto en el hogar como en las empresas, al utilizar solo los más necesarios para la vida diaria.

Reutilizar: extender el uso de dichos aparatos antes de desecharlos, mientras aún funcionen completa o parcialmente. Estos se pueden reparar, vender, donar o intercambiar por otros.

Reciclar: procurar la actualización de dichos aparatos mediante fabricantes que dan la opción de recibir los viejos modelos como parte de pago. Así, dichos fabricantes, u otras empresas de reciclaje, se encargan de descomponer los AEE en piezas que puedan reutilizarse o en materiales que sirvan como materia prima para fabricar nuevos productos.

Se puede incluso considerar una erre más: recuperar. En un sentido más amplio al de reutilizar, esta última promueve el uso de los materiales reciclados en la fabricación de objetos funcionalmente diferentes, hecho que permite pensar la creación de empresas distintas (Borrás, 2018). Así, en este trabajo se consideró esta otra erre al emplear materiales reciclados de AEE para elaborar nuevos utensilios que cumplan funciones muy diferentes a las establecidas en su diseño original y que puedan ser un potencial origen de microempresas.

Para justificar la necesidad de reciclar los AEE, el líder de la gestión ambiental de Une, José Jaime Restrepo, señala que "cuando llegan a un relleno sanitario inadecuado, al suelo o a una quebrada, los equipos electrónicos pueden lixiviar [filtrar] metales pesados, que pueden llegar al agua, al aire o a los animales" (Peñarredonda, 2013).

En cuanto al éxito en este proceso, dicho funcionario dice que del $35 \%$ al $40 \%$ de los equipos electrónicos logran reutilizarse, y que logran recuperarse hasta el $92 \%$ de los materiales reciclados, para finalmente desechar apropiadamente los residuos que no pueden aprovecharse (Peñarredonda, 2013).

\section{Empresas de reciclaje electrónico en Colombia}

\section{Pilas Colombia}

"Pilas con el Ambiente" es el programa de recolección de pilas usadas liderado por la Andi, cuyo objetivo principal es cerrar el ciclo de vida del producto de manera conjunta con el consumidor y beneficiar así al medio ambiente. Los miembros de este sistema son: Almacenes Éxito S. A., Coéxito S. A., Electrón Colombia S. A. S.,
Energía Integral Andina S. A., Eveready de Colombia S. A., Foto del Oriente Ltda., General Motors Colmotores S. A., Importadora AMG S. A. S., Nacional de Pilas Occidente S. A. S., Procter \& Gamble Ltda., Spectrum Brands Colombia S. A., Starkey Laboratories Colombia Ltda. (Pilas Colombia, 2010). 


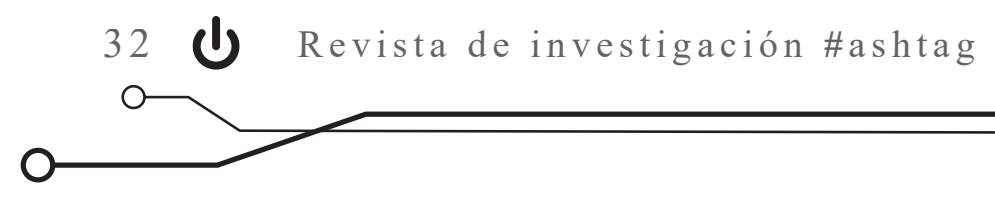

\section{EcoCómputo}

EcoCómputo está conformado por varias empresas cuyo propósito es recolectar residuos de aparatos eléctricos y electrónicos (RAEE), en particular, provenientes de equipos computacionales. Este colectivo surgió frente a la gestión del Gobierno Nacional, en procura de la

\section{Lúmina}

$\mathrm{Al}$ contrastar los bombillos, o lámparas incandescentes, con las lámparas fluorescentes compactas (LFC), se tiene como desventaja que estas últimas contienen una pequeña cantidad de mercurio, por lo cual requieren de un adecuado proceso de reciclaje (DesignBlog, 2011). Lúmina está comprometida con el total aprovechamiento

\section{Click on Green}

Esta empresa nació con el interés de atender el problema del inadecuado tratamiento de la basura electrónica, al considerar que el 90 \% de los materiales en los RAEE es recuperable como materia prima en la industria. Por ello, se ha comprometido con disponer apropiadamente de los responsabilidad social empresarial, para darle un manejo adecuado a dicho tipo de residuos en Colombia y cumplir con la Resolución 1512 de 2010, del Ministerio de Ambiente y Desarrollo Sostenible (MADS) (Velásquez, 2016).

de los residuos de las bombillas eléctricas, de conformidad con la Resolución 1511 de agosto de 2010 del Ministerio de Ambiente, Vivienda y Desarrollo Territorial, ahora Ministerio de Ambiente y Desarrollo Sostenible, y del Decreto 4741 de 2005 (Sánchez, 2016).

componentes propios de equipos computacionales y periféricos. Para este fin, emplea altos estándares de calidad y seguridad, y garantiza el borrado de información confidencial (Click on Green, 2012).

\section{Universidades que manejan reciclaje electrónico}

Pese a que en Bogotá hay al menos unas diez instituciones de educación superior que tienen programas de ingeniería electrónica -en cierta forma, semejantes al que se oferta en la CuN-, solo se encontró evidencia de reciclaje electrónico en una universidad. El proyecto de reciclaje electrónico de la Universidad Nacional de Colombia pretende realizar una conexión directa con las empresas que manejan equipos electrónicos y concientizarlas del gran beneficio ambiental que conlleva el manejo apropiado de los residuos electrónicos, además del potencial beneficio económico que esto significa para las empresas y sus trabajadores (Unimedios, 22 de julio de 2016). 


\section{Desarrollo del programa de reciclaje en la cun}

Con la finalidad de concientizar a la comunidad del programa de Ingeniería Electrónica de la cuN sobre la importancia y los potenciales beneficios de hacer reciclaje electrónico, se organizó una Reciclatón. Este evento se planeó para recolectar desechos eléctricos y electrónicos. En la figura 1 se muestra el respectivo afiche publicitario. A través del correo electrónico y la promoción directa en cada salón de clase, se informó a los estudiantes sobre el programa de reciclaje electrónico organizado por el semillero de investigación ElectroCUN.

Figura 1. Publicidad de la Reciclatón

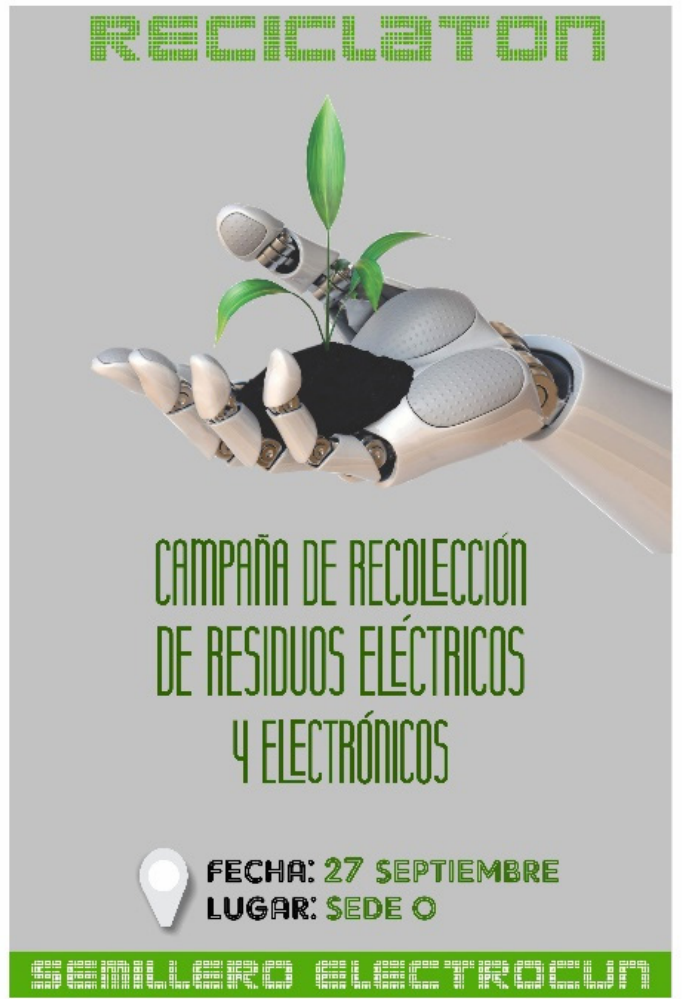

Fuente: elaboración propia

El punto de recolección se ubicó en la Sede O de la cun, tal como se detalla en la figura 2. Tras constatar la intención de algunos estudiantes de aportar más elementos eléctricos y electrónicos, el plazo inicialmente establecido para esta recolección debió extenderse.

Figura 2. Jornada de recolección de RAEE en la CUN

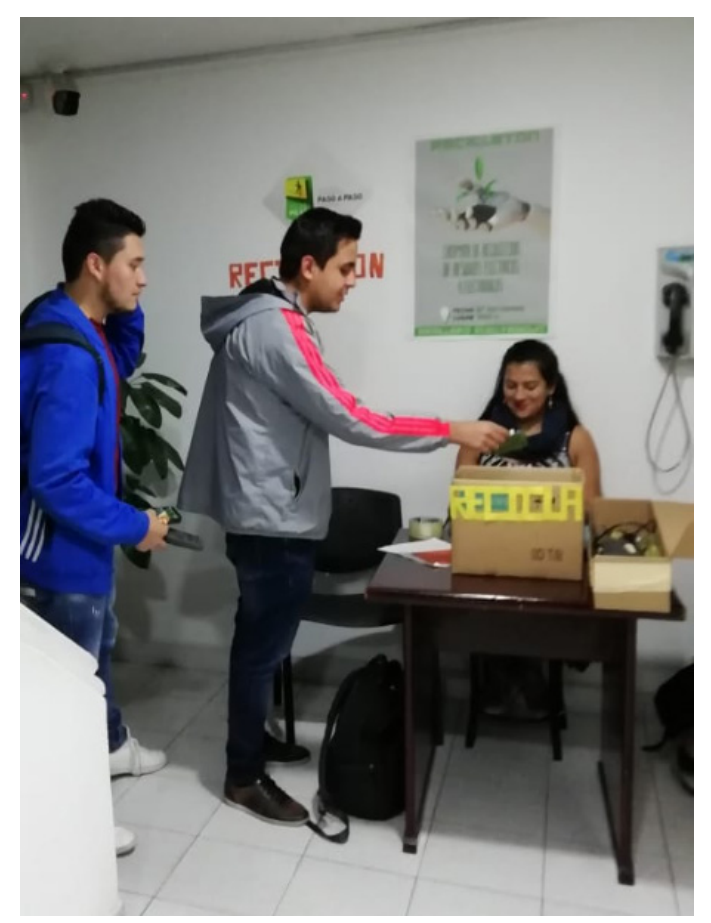

Fuente: elaboración propia

Tras la Reciclatón, se catalogaron los componentes recolectados en funcionales y no funcionales. La clasificación de los componentes funcionales exigió una revisión técnica, en particular de los motores (Electrotec, 6 de noviembre de 2014; Higinio, 9 de septiembre de 2016) y las fuentes de PC (DaeNET, 2009; Joshuamarius, 11 de octubre de 2009). Esto con el propósito de establecer un sistema de dispensación que facilite, en calidad de préstamo, componentes eléctricos y electrónicos funcionales a los estudiantes para su uso apropiado en el desarrollo de proyectos académicos. 
En cuanto a los componentes no funcionales, se proyectó un plan de recuperación (Borrás, 2018) mediante la elaboración de artesanías. Esta estrategia se diseñó con la idea de crear una posible microempresa y también para ejemplificar su uso en actividades de expresión artística en colegios de niños con bajos recursos económicos.

\section{Resultados obtenidos}

En la tabla 1 se presenta el inventario del mate-

parte de su clasificación en componentes funciorial reciclado y, en las figuras 3,4 y 5 , se muestra nales y no funcionales.

Tabla 1. Inventario de los componentes reciclados

\begin{tabular}{|l|c|}
\hline \multicolumn{1}{|c|}{ Descripción } & Cantidad \\
\hline Probador de red & 1 \\
\hline Cables de poder & 5 \\
\hline Fusibles & 4 \\
\hline Puente rectificador & 4 \\
\hline Bus ID & 2 \\
\hline Mouse & 1 \\
\hline Cable de línea telefónica & - \\
\hline Alambre uTP & - \\
\hline 74LS245 & 2 \\
\hline Transformador & 2 \\
\hline Cable dúplex & - \\
\hline Baquela & 3 \\
\hline Jack hembra RJ45 & 1 \\
\hline Jack hembra teléfono & 1 \\
\hline Parlantes & 4 \\
\hline Matriz numérica & 1 \\
\hline Ventilador 5 V & 8 \\
\hline Bocina & 4 \\
\hline Motor paso a paso & 2 \\
\hline Modulo TTL & varias \\
\hline Resistencias & \\
\hline Condensadores & \\
\hline Fuentes & \\
\hline Motores DC & \\
\hline Relés & \\
\hline
\end{tabular}

Fuente: elaboración propia 
Figura 3. Cable clasificado

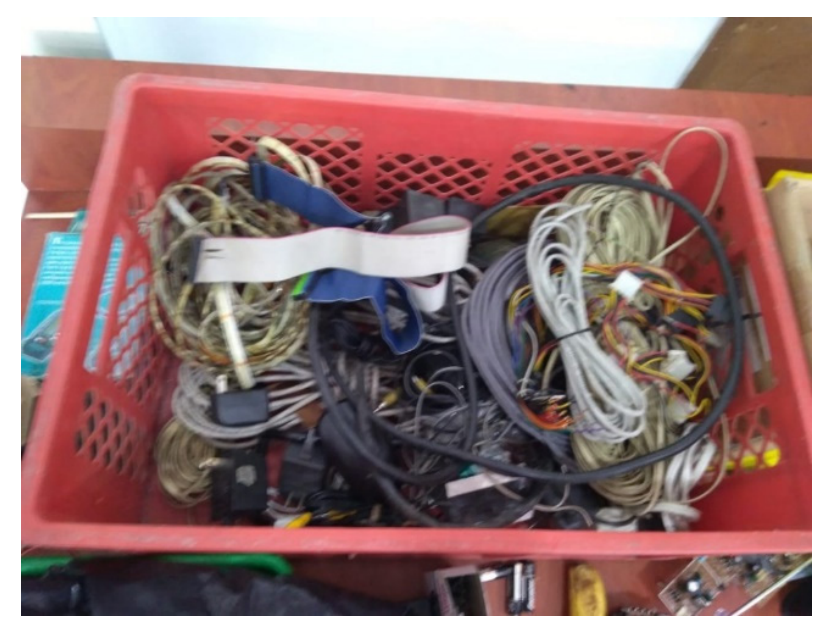

Fuente: elaboración propia

Figura 4. Componentes reutilizables

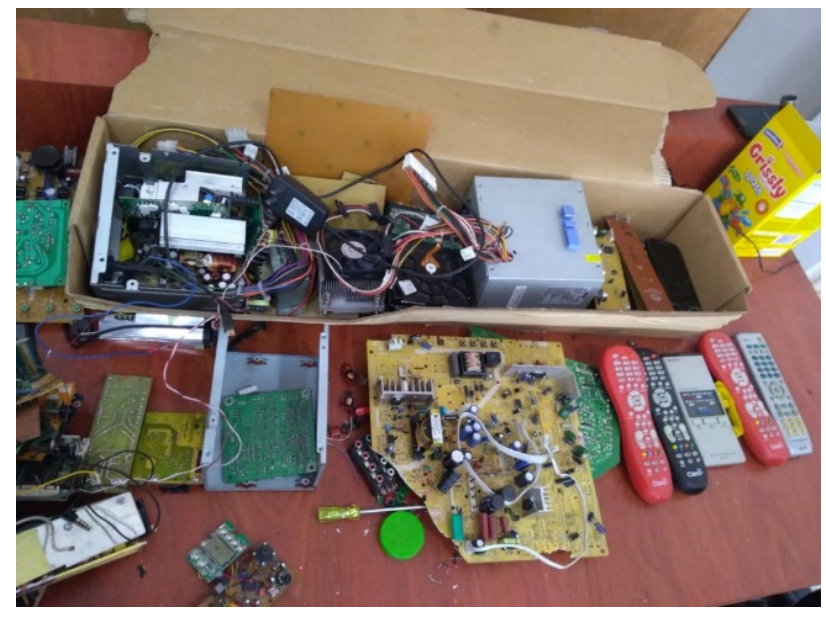

Fuente: elaboración propia

\section{Componentes funcionales}

Las piezas de equipos computacionales e impresoras, como fuentes de alimentación, ventiladores y motores paso a paso, entre otros, fueron probadas y validadas como totalmente funcionales. Estas se dejaron intactas y a disposición de los estudiantes para el desarrollo de sus proyectos. Elementos como cables y conectores se dejaron en un espacio aparte, puesto que este material no es para préstamo sino para su uso final en el montaje de circuitos.
Este programa de reciclaje electrónico ha demostrado tener un gran impacto en los estudiantes, puesto que ellos tendrán la posibilidad de adquirir material que les ayude en la implementación de sus proyectos. Este quedó disponible en el laboratorio, para ser prestado en cualquier momento de la jornada académica. 


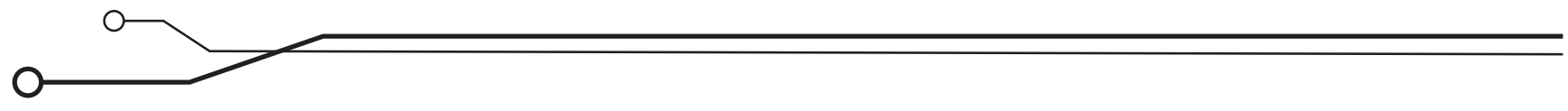

\section{Componentes no funcionales}

Estos otros componentes electrónicos se utilizaron para elaborar artesanías y decoraciones, tales como bisutería y robots figurativos, lo que permitió su recuperación (Borrás, 2018) en una utilidad diferente y creativa.

Figura 5. Componentes para manualidades

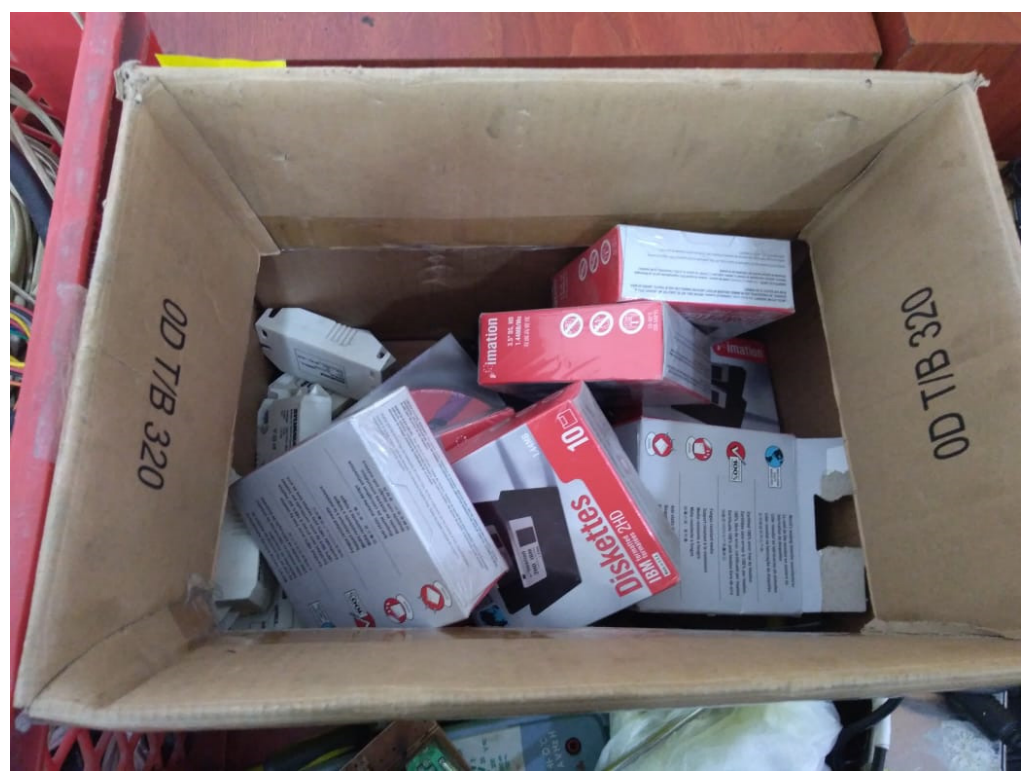

Fuente: elaboración propia

\section{Elaboración de artesanías}

En la figura 6 se detalla un ejemplo de bisutería diseñada a partir de componentes electrónicos. Recientemente, se ha visto una gran cantidad de emprendimientos que usan libre y creativamente resistencias, condensadores, circuitos impresos, leds y transistores, orientados a fundar microempresas basadas en esta idea. En la figura 7 se detalla un ejemplo de expresión artística que puede explotarse en contextos escolares, tanto como escena estática como con la posibilidad de realizar animaciones de stop-motion. De esta manera, pueden recuperarse los componentes electrónicos no funcionales.
Figura 6. Bisutería con resistencias

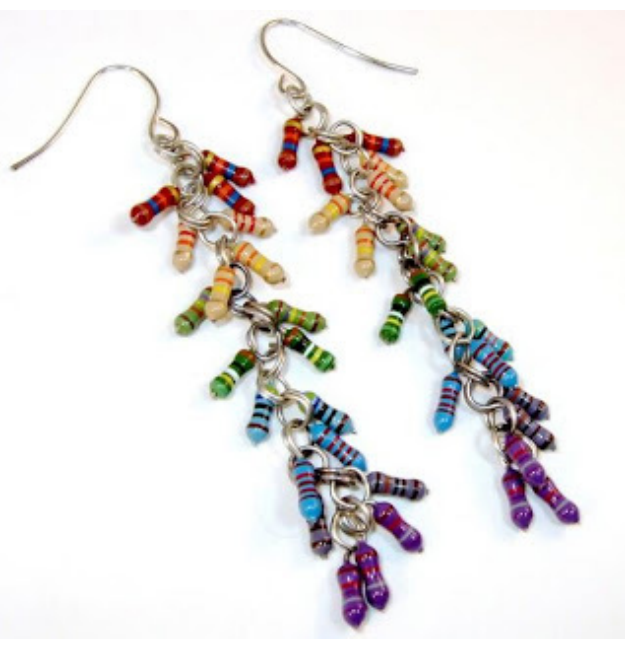

Fuente: Carmenjuarros2000 (2013, párr. 4) 
Figura 7. Escena de personajes, realizada con varios elementos electrónicos

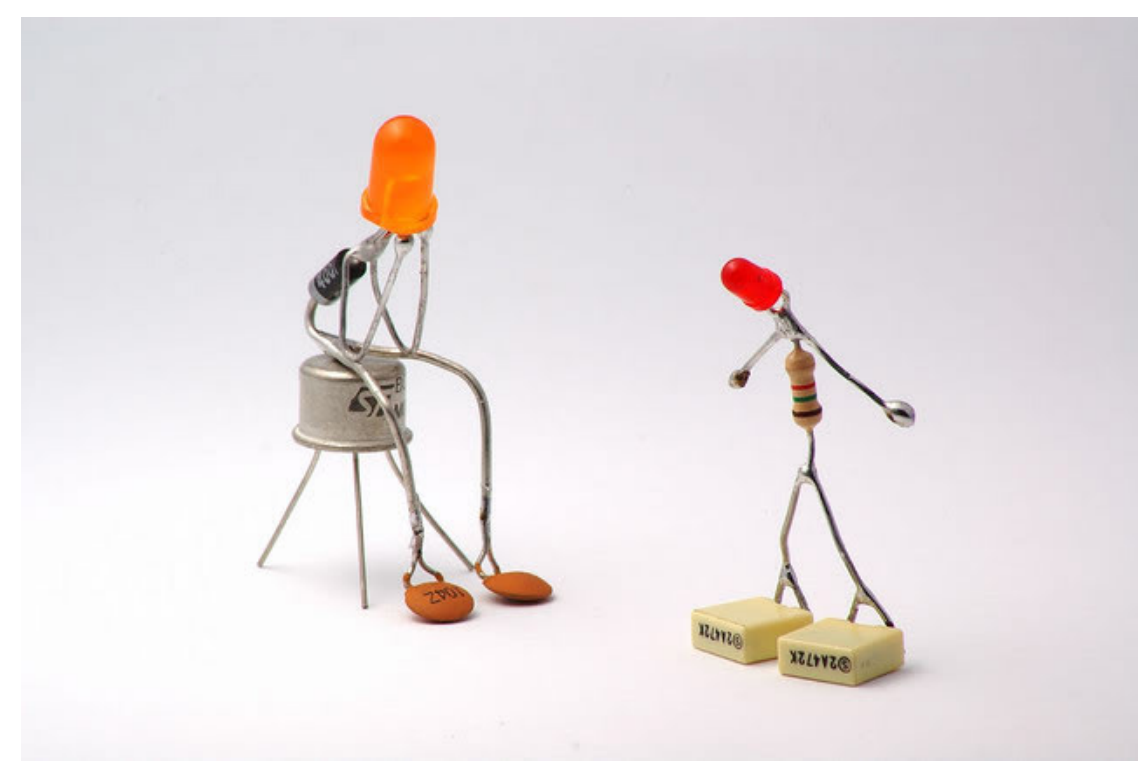

Fuente: Robots S. A. de C. V. (2013, párr. 1)

Adicionalmente, la figura 8 muestra dos robots figurativos, realizados para este artículo, que

\section{Sugerencias de los estudiantes}

Los estudiantes fueron encuestados y realizaron algunas sugerencias para el mejoramiento del programa del reciclaje electrónico. Entre ellas están:

- Extender la recolección de RAEE a todas las sedes de la cun.

- Hacer más jornadas tipo Reciclatón para que más estudiantes participen.

- Publicitar mejor el evento y ofrecer más tiempo para la recolección. emplean material eléctrico y electrónico no funcional recolectado durante la Reciclatón.
- Permitir que otros estudiantes continúen el programa de reciclaje como opción de grado I o II.

- Permitir que los estudiantes propongan otras iniciativas semejantes a este programa de reciclaje, que propendan por la conservación y protección del medio ambiente.

- Ofrecer incentivos por colaborar con la entrega del material. 


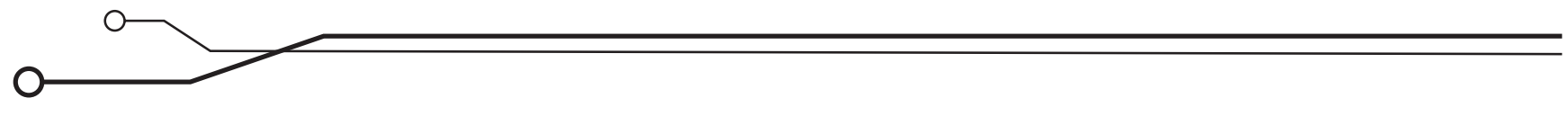

Figura 8. Robots figurativos hechos con materiales eléctricos y electrónicos no funcionales
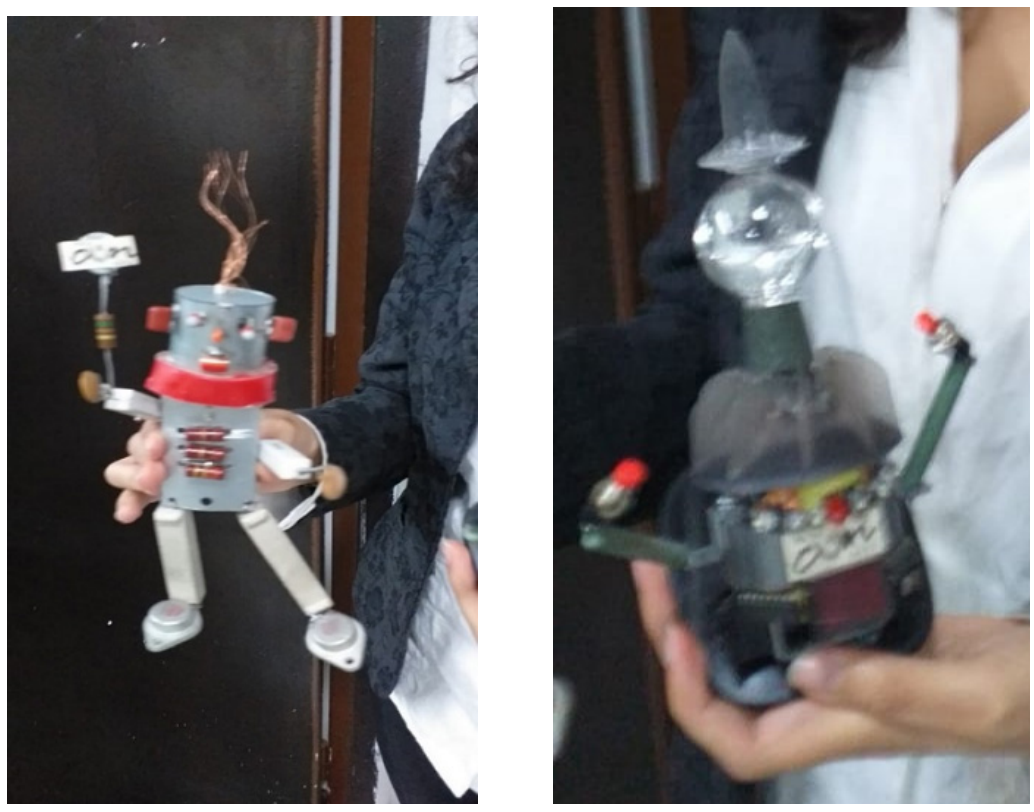

Fuente: elaboración propia

\section{Resultados de la encuesta}

La figura 9 indica que menos de la mitad de los estudiantes encuestados tenían conocimiento del programa de reciclaje electrónico. Por ello, la figura 10 indica que faltó informar más a los estudiantes sobre dicho programa. La figura 11 indica que prácticamente la mitad de los estudiantes encuestados participó en la recolección de los artefactos eléctricos y electrónicos para reciclar.

La figura 12 indica que un poco más de la mitad de los estudiantes tenía conocimiento sobre los beneficios que trae para la CUN desarrollar dicho programa. Por su parte, la figura 13 indica que bastantes estudiantes lograron conocer los beneficios que dicho programa les trae, como apoyo a sus estudios. La figura 14 indica que la mayoría de los estudiantes están de acuerdo con realizar otra jornada de reciclaje electrónico para participar en ella. Finalmente, la figura 15 indica que esta actividad tuvo un gran impacto, pues casi todos los estudiantes la encontraron muy útil.

Figura 9. ¿Conoce sobre el programa de reciclaje electrónico que se desea implantar en la cun?

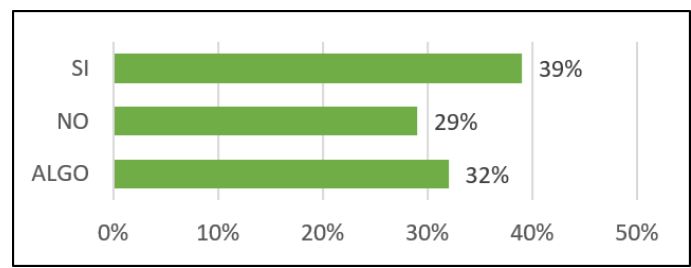

Fuente: elaboración propia 
Figura 10. ¿Faltó más información sobre dicho programa y la jornada de recolección de artefactos eléctricos y electrónicos para reciclar?

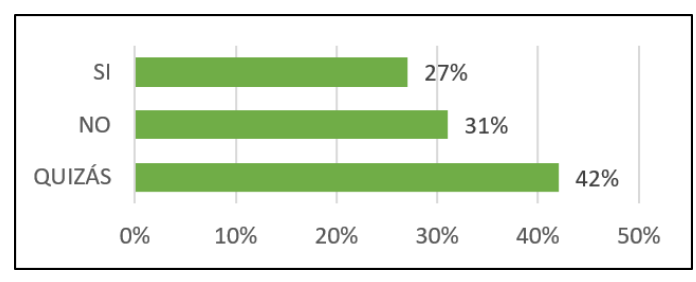

Fuente: elaboración propia

Figura 11. ¿Participó en la Reciclatón?

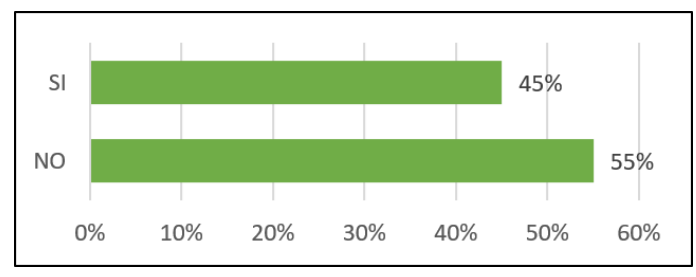

Fuente: elaboración propia

Figura 12. ¿Conoce los beneficios del programa de reciclaje electrónico para la cun?

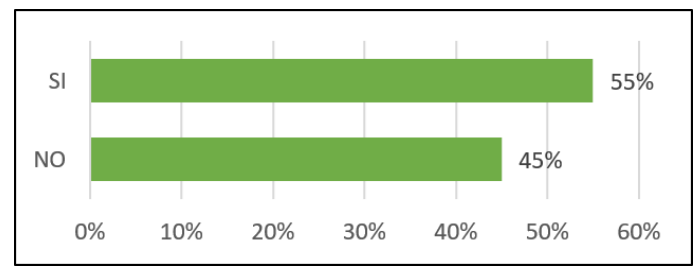

Fuente: elaboración propia

Figura 13. ¿Conoce los beneficios de dicho programa para usted como estudiante de la cun?

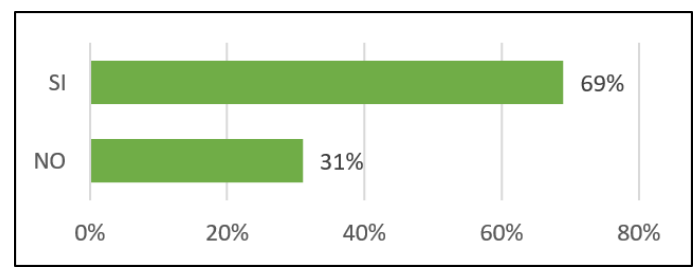

Fuente: elaboración propia 


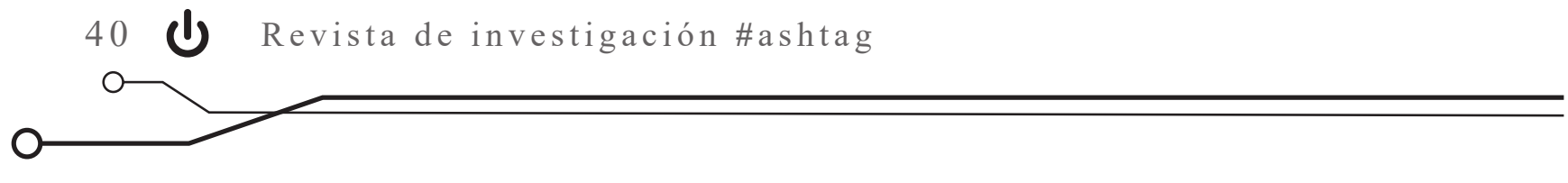

Figura 14. ¿Participaría nuevamente en una futura jornada de recolección de AEE para reciclar?

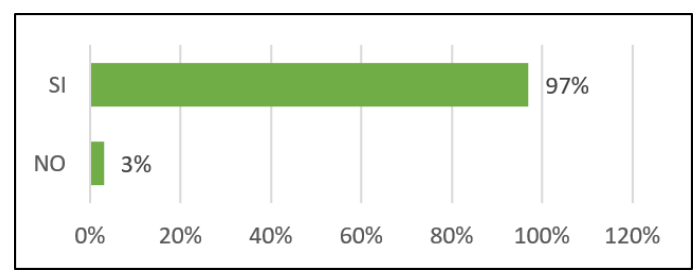

Fuente: elaboración propia

Figura 15. ¿Cuál es su opinión general sobre la actividad de reciclaje realizada en la Sede O?

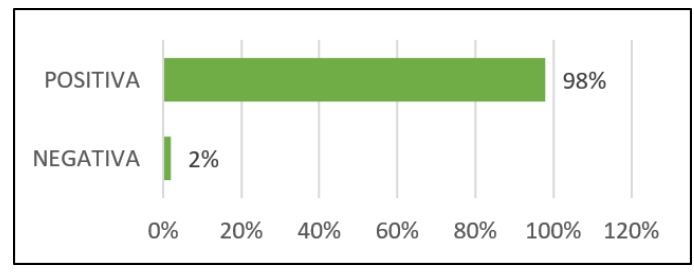

Fuente: elaboración propia

\section{Conclusiones}

Se dio a conocer a la comunidad cunista del programa de Ingeniería Electrónica sobre la idea de establecer una actividad de reciclaje electrónico, impulsada por el semillero de investigación ElectroCUN. Como un primer paso, se logró realizar una Reciclatón, publicitada con carteles informativos, correos masivos y promoción en cada uno de los salones y laboratorios de la Sede O.

La jornada de recolección de artefactos eléctricos y electrónicos para reciclar -realizada entre el 27 y el 29 de Septiembre de 2018- logró un primer repositorio significativo de elementos funcionales que estará a disposición de los estudiantes para su reutilización en el desarrollo de proyectos académicos.

Por su parte, el material no funcional se destinó a recuperarse mediante alternativas que impactarían en aspectos propios de proyección social y responsabilidad ecológica. Varios meses después de la Reciclatón, algunos estudiantes evidenciaron interés en seguir apoyando esta iniciativa con la donación de más residuos eléctricos y electrónicos para reciclar.

La encuesta realizada a algunos estudiantes evidenció gran participación e interés por el proyecto. Este hecho mejoró la socialización de la actividad de reciclaje realizada $\mathrm{y}$, adicionalmente, permitió conocer diferentes opiniones y aspectos por mejorar de cara a la continuación del programa de reciclaje electrónico.

Tras la clasificación de los componentes ya reciclados y su almacenaje en un armario especialmente destinado a su dispensación, queda como trabajo futuro automatizar las gavetas de dicho 
armario, de tal manera que, al necesitarse cierto componente, se ilumine un indicador led para saber rápidamente en dónde está ubicado.

Como los componentes no funcionales pueden ser usados para manualidades, queda como trabajo futuro establecer una jornada pedagógica con estudiantes de colegio para dar a conocer un poco sobre los elementos electrónicos y sobre el programa de reciclaje electrónico que se está implementando en la CUN.

Se espera que este programa de reciclaje siga fortaleciéndose para evaluar y promover el uso del material reciclado por parte de los estudiantes.

\section{Referencias}

Borrás, C. (2018). Las 4R del reciclaje [entrada de blog]. Ecología Verde. Recuperado de https:// www.ecologiaverde.com/las-4r-del-reciclaje-421.html

Carmenjuarros2000. (11 de abril de 2013). Artículos con reciclaje [entrada de blog]. Recuperado de https:/ / carmenjuarros2000.blogspot.com/2013/04/articulos-con-reciclaje.html

Click on Green. (2012). Reciclaje responsable de electrónicos [recurso en línea]. Recuperado de https://www.clickongreen.com/

DaeNET. (2009). Probar fuentes de poder sin PC [recurso en línea]. Recuperado de https:/ / daenet. wordpress.com/2009/07/02/probar-fuentes-de-poder-sin-pc/

DesignBlog (2011). Lámparas fluorescentes compactas (LFC) [recurso en línea]. Recuperado de http:/ / designblog.uniandes.edu.co/blogs/dise2308/files/2011/09/documento_productolite4. pdf

Electrotec. (6 de noviembre de 2014). Prueba de motores Dc pequeños [archivo de video]. Recuperado de https:/ / www.youtube.com/watch?v=SJ36YTYCMZ4

Higinio, H. (9 de septiembre de 2016). Motor de Pasos -Stepper Motor-. ¿Cómo probarlo e identificar sus bobinas? Parte2 [archivo de video]. Recuperado de https:/ / www.youtube.com/ watch?v=7-t7tWGeUXg

Joshuamarius. (11 de octubre de 2009). La computadora saludable 2. La fuente de poder o power supply [archivo de video]. Recuperado de https://www.youtube.com/watch?v=iLOUziv0cGE

Peñarredonda, J. (2013). ¿Dónde y por qué reciclar los residuos electrónicos? [entrada de blog] Enter.co. Recuperado de https://www.enter.co/cultura-digital/ciudadinteligente/ donde-y-por-que-reciclar-los-residuos-electronicos / 


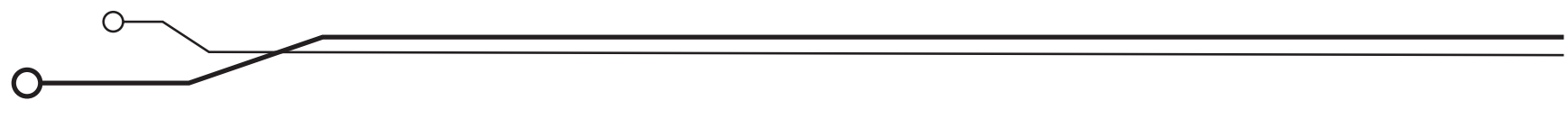

Pilas Colombia. (2010). Programa "Pilas con el Ambiente" [recurso en línea]. Recuperado de https://www.pilascolombia.com

Responsabilidad Social Empresarial y Sustentabilidad. (2014). 3R. La regla de las tres erres (reducir, reciclar y reutilizar) [entrada de blog]. Recuperado de https:/ / www.responsabilidadsocial.net/3r-la-regla-de-las-tres-erres-reducir-reciclar-y-reutilizar/.

Robots S. A. de C. V. (2013). Productos [entrada de blog]. Recuperado de http://robotssa. blogspot.com/2013/02/productos.html

Sánchez, M. (2016). Identificación de la situación actual manejo y la gestión de los residuos de aparatos eléctricos y electrónicos RAEE en la zona urbana del municipio de Cajicá [trabajo de grado]. Universidad de Ciencias Aplicadas y Ambientales (UDCA), Bogotá, Colombia.

Unimedios. (22 de julio de 2016). Estudiantes impulsan reciclaje de aparatos eléctricos. Recuperado de http://agenciadenoticias.unal.edu.co/detalle/article/estudiantes-impulsan-reciclaje-de-aparatos-electricos.html

Velásquez, A. (2016). Investigación exploratoria sobre generación y gestión de residuos hospitalarios tipo RAEE en Bogotá D. C. Bogotá: Universidad Nacional Abierta y a Distancia (UNAD). 